\title{
FDA PUBLIC ADVISORY FOR NEVIRAPINE (VIRAMUNE)
}

Recently the US Federal Drug Administration has issued a Public Advisory for Nevirapine (NVP) (Vira-mune) informing health care providers of a safety-related change to the nevirapine package insert. This advisory, issued on 19 January 2005, notified a change in nevirapine prescribing information with a warning against starting nevirapine in women with CD4 counts $>250$ cells/ $\mu$ land in men with counts $>400 / \mu l$. The risk of developing a hepatic hypersensitivity reaction in the first 6 weeks of nevirapine therapy had been shown to be increased 12-fold in women with counts $>250 / \mu l$ and 5 -fold in men with counts $>400 / \mu$, compared with women with counts $<250 / \mu \mathrm{l}$ and men with counts $<$ $400 / \mu \mathrm{l}$.

Nevirapine was the first non-nucleoside drug (NNRTI) to be approved by the Federal Drug Administration (FDA) for use in highly active antiretroviral therapy (HAART) of HIV infection in 1996. Since then it has been extensively used throughout the world, and it is manufactured in a very inexpensive generic form and in FIXED-DOSE combinations. It is also licensed for use in prevention of mother-to-child transmission.

That this warning should come to light at this stage is probably due to a number of factors which will be reviewed by a senior HIV specialist in the next edition of this journal. In this review, recommendations for the use of nevirapine in the public and private sectors are made.

The toxicity strongly associated with NVP is hypersensitivity hepatitis characterised by rash, raised transaminases (> $5 \times$ normal) or systemic illness and occurs within 42 days of commencement. The risk for this reaction is modified by gender and CD4 count.

These warnings will have implications for commencement of HAART in patients with higher CD4 counts. This is most likely to be pregnant women. The warning will also apply to patients switching later in their course of HAART when CD4 counts have improved, again most likely due to pregnancy or in those women wishing to conceive. In the private sector there are more choices, since other options such as nelfinavir exist. In the national roll-out plan it may be necessary to anticipate fertility issues and initiate treatment with NVP in these women when CD4 counts are still low; if a change is needed later in treatment when CD4 counts are higher, utilisation of the second-line drug. Kaletra may be required.

We print the FDA warning below, but look out for a South African expert's perspective on the public advisory and the other publicity surrounding this drug in the next edition of this Journal.

The editors

FDA PUBLIC HEALTH ADVISORY FOR NEVIRAPINE (VIRAMUNE)

\section{http://www.fda.gov/cder/drug/advisory/} nevirapine.htm

This public health advisory informs health care providers and patients about recent safety-related changes to the nevirapine (Viramune) label (package insert) and about appropriate use of HIV triple combination therapy containing nevirapine, which is one treatment option in the United States and which is increasingly being used globally. The nevirapine label has been revised several times over the last two years to include more information on liver toxicity associated with long term nevirapine use. The Indications and Usage section of the Viramune label now recommends against starting nevirapine treatment in women with CD4+ cell counts greater than 250 cells $/ \mathrm{mm}^{3}$ unless benefits clearly outweigh risks. This recommendation is based on a higher observed risk of serious liver toxicity in patients with higher CD4 cell counts prior to initiation of therapy. In addition, the revised label now includes a Medication Guide to inform patients about risks associated with nevirapine when used for the treatmentofHIV. Both clinically symptomatic and asymptomatic liver toxicity are observed with long-term use of nevirapine in combination with other HIV drugs. Asymptomatic liver toxicity is defined as increases in liver enzymes without any associated clinical signs or symptoms and is similar to that seen with other antiretroviral drugs. Symptomatic liver toxicity is more common with nevirapine compared to other antiretroviral drugs. Important information regarding symptomatic nevirapine liver toxicity is summarised as follows: 
Symptomatic nevirapine liver toxicity consists of elevated liver enzymes plus at least one symptom, which is typically rash but may include flu-like symptoms or fever. The severity of symptomatic liver toxicity ranges from mild symptoms with liver enzyme abnormalities to rapidly occurring liver failure and death.

- Symptomatic nevirapine liver toxicity typically occurs after only a few weeks of dosing and may progress to liver failure despite monitoring of laboratory tests, which is not characteristic of other antiretrovirals.

- Females and patients with higher CD4+ cell counts are at increased risk of liver toxicity. Females have a threefold higher risk of symptomatic nevirapine liver toxicity than males, and females with CD4+ cell counts > 250 cells $/ \mathrm{mm}^{3}$ have a 12-fold higher risk of symptomatic liver toxicity than females with CD4+ cell counts $<250$ ( $11 \%$ v. $0.9 \%)$. Males with CD4+ cell counts $>400$ cells $/ \mathrm{mm}^{3}$ have a five-fold higher risk of symptomatic liver toxicity than males with CD4+ cell counts $<400$ (6.3\% v.1.2\%).

- Nevirapine-related deaths due to symptomatic liver toxicity, including some in HIV-infected pregnant women, have been reported to FDA's Medwatch program. Serious and fatal liver toxicity has not been reported after single doses of nevirapine.

In spite of the potential for serious and lifethreatening liver toxicity and skin rashes with nevirapine, there are multiple reasons why nevirapine remains an important part of an HIV treatment regimen for many HIV-infected individuals worldwide. These reasons include:

- Triple antiretroviral regimens have been shown to have a large impact on the reduction of AIDS morbidity and mortality. Triple antiretroviral drug regimens containing a protease inhibitor (PI) or a non-nucleoside reverse transcriptase inhibitor (NNRTI), such as nevirapine, are standard of care for HIV treatment and are needed to adequately and durably suppress virus.
Many options are needed for HIV-infected patients since resistance to antiretroviral drugs or to an entire antiretroviral class can develop.

- Symptomatic liver toxicity has not been reported with the use of single doses of nevirapine to the mother and to the child for prevention of perinatal HIV infection.

- Alternatives to nevirapine are limited by other toxicities, potential drug interactions, and by the risk of drug-related birth defects if given to a female in the first trimester of pregnancy.

- Nevirapine liver toxicity is less frequent $(<2 \%$ for both males and females with CD4+ cell counts $<250$ cells $/ \mathrm{mm}^{3}$ ) when started in patients with lower CD4 counts. Therefore, symptomatic liver toxicity in resource poor countries is likely to be much lower if World Health Organization standards are used for starting treatment. The WHO recommends the initiation of ART treatment in patients with advanced disease or with $\mathrm{CD} 4$ counts $<200$ cells $/ \mathrm{mm}^{3}$.

- Nevirapine is chemically stable in environmental conditions where other antiretrovirals are not.

- Symptomatic liver toxicity has not been reported in HIV-infected children, and nevirapine is available in a liquid formulation while many other antiretrovirals are not.

In conclusion, the seriousness of the underlying disease must be considered as part of the risk benefit analysis when treating HIV-infected patients. HIV infection will progress to AIDS and death if untreated. Treatment with combination antiretroviral drugs, including nevirapine, can slow clinical progression and may delay the development of AIDS or death for years. Health care providers should weigh the benefits and risks associated with nevirapine use before prescribing nevirapine for the treatment of their HIV-infected patients.

Date created: 19 January 2005 